Santa Clara University

Scholar Commons

Psychology

College of Arts \& Sciences

2012

\title{
Assessing metacognitive skills in waking and sleep: A psychometric analysis of the metacognitive, affective, cognitive experience (MACE) questionnaire
}

Tracey L. Kahan

Kieran T. Sullivan

Santa Clara University, ksullivan@scu.edu

Follow this and additional works at: http://scholarcommons.scu.edu/psych

Part of the Psychology Commons

\section{Recommended Citation}

Kahan, T.L. \& Sullivan, K.T. (2012). Assessing metacognitive skills in waking and sleep: A psychometric analysis of the metacognitive, affective, cognitive experience (MACE) questionnaire. Consciousness and Cognition, 20, 340 - 352.

NOTICE: this is the author's version of a work that was accepted for publication in Consciousness and Cognition. Changes resulting from the publishing process, such as peer review, editing, corrections, structural formatting, and other quality control mechanisms may not be reflected in this document. Changes may have been made to this work since it was submitted for publication. A definitive version was subsequently published in Consciousness and Cognition, Vol. 21, no. 1, (2012).

doi:10.1016/j.concog.2011.11.005.

This Article is brought to you for free and open access by the College of Arts \& Sciences at Scholar Commons. It has been accepted for inclusion in Psychology by an authorized administrator of Scholar Commons. For more information, please contact rscroggin@scu.edu. 
Assessing Metacognitive Skills in Waking and Sleep: A Psychometric Analysis of the Metacognitive, Affective, Cognitive Experience (MACE) Questionnaire

Tracey L. Kahan ${ }^{\mathrm{a}}$ and Kieran T. Sullivan ${ }^{\mathrm{a}}$ Santa Clara University

a Department of Psychology, Santa Clara University, Santa Clara, CA 95053, United States

Correspondence concerning this article should be addressed to Tracey L. Kahan, Department of Psychology, Santa Clara University, 500 El Camino Real, Santa Clara, CA 95053, United States E-mail: tkahan@scu.edu.

KEYWORDS: high-order cognition, metacognition, reflective awareness, assessment, cross-state cognition.

HIGHLIGHTS (see guidelines on website of Consciousness and Cognition)[max 85 chars each] 1. Psychometric analysis of a measure of metacognition appropriate for use across conscious states.

2. The Metacognitive, Affective, Cognitive Experience (MACE) Questionnaire is valid and reliable.

3. The factor structure of the MACE includes one control and two monitoring factors.

4. The psychometric properties of the MACE replicate and extend the Metacognitive Model. 


\begin{abstract}
The Metacognition, Affective, Cognitive Experience (MACE) questionnaire was designed to assess metacognition across sleep and waking (Kahan \& LaBerge, 1996). The present research evaluates the psychometric properties of the MACE. Data from two recent studies $(N=185)$ were used to assess the inter-item consistency, test-retest reliability, and factorial, convergent, and discriminant validity of the MACE. Results show that the MACE is a reliable measure with good construct validity. Exploratory factor analyses revealed one self-regulation and two monitoring factors. One monitoring factor emphasized monitoring internal conditions; the other emphasized monitoring external conditions. This factor structure is consistent with the Metacognitive Model (Nelson \& Narens, 1990). Tests of convergent and discriminant validity suggest that the MACE is assessing metacognition and is appropriately related to similar constructs such as mindfulness and self-consciousness. The implication of these findings as well as suggestions for research and clinical applications of the MACE are discussed.

[149 words]
\end{abstract}


Assessing Metacognitive Skills in Waking and Sleep: A Psychometric Analysis of the

Metacognitive, Affective, Cognitive Experience (MACE) Questionnaire

"Introspective observation is what we have to rely on first and foremost and always."

William James (Principles of psychology, p. 185)

\section{Introduction}

The purpose of the current research is to examine the psychometric properties of a measure of metacognition intended for use across conscious states (i.e. sleeping and waking). Metacognition refers to the knowledge, understanding, and regulation of one's own cognitive processes (Flavell, 1979; Nelson \& Narens, 1990). Examples of metacognition include knowledge of which memory strategies are most useful in specific learning situations, the realization that reading speed must be reduced to understand a difficult passage, and electing to focus on a friend's voice to better understand the story he is telling.

\subsection{Why is metacognition important?}

Metacognition has been of particular interest to cognitive, developmental, educational, social, and clinical psychologists (Dimaggio \& Lysaker, 2010; Metcalfe \& Shimamura, 1994; Perfect \& Schwartz, 2002; Semerari et al., 2003). Metacognitive skills are critical to learning-tolearn (Brown, Campione, \& Day, 1981), error correction (Fernandez-Duque, Baird, \& Posner, 2000; Nelson, 1996), source monitoring (Johnson, Hashtroudi, \& Lindsay, 1993), the development and utilization of expertise (Garner \& Alexander, 1989); emotion regulation (Posner \& Rothbart, 1998), and the ability to take another person’s point of view (“theory of mind”)(Flavell, 2000; Schneider \& Lockl, 2008). Metcalfe (2008, pp. 29-31) makes the point that the type of self-reflective consciousness involved in metacognition conferred an important evolutionary advantage, allowing one "to reflect on their actions and their outcomes and change 
those actions as indicated by the reflection to obtain better results" (than those without this capacity)(also see Ardila, 2008).

As described by Nelson and Narens (1994),

"Metacognition is simultaneously a topic of interest in its own right and a bridge between areas, e.g., between decision making and memory, between learning and motivation, and between learning and cognitive development” (p. 1).

\subsection{Theoretical framework: Nelson and colleagues’ Metacognitive Model}

According to the Nelson and Narens (1990; 1994) Metacognitive Model, metacognition involves an interaction between two levels of mental representations, the object-level and the meta-level (Figure 1). Information about an individual's current, ongoing experience (objectlevel) is continuously conveyed to the meta-level via monitoring processes, which include noticing and evaluating current knowledge, understanding, awareness, and emotion (Fernandez-Duque et al., 2000; Metcalfe, 2008). Information about behavior change (i.e., what to do next) is conveyed from the meta-level to the object-level via control processes, which include efforts to regulate one’s thoughts, feelings, or behavior in relation to a goal or intention (Mazzoni \& Nelson, 1998; Nelson, 1996; Son \& Schwartz, 2002).

For example, consider the remark: "I was thinking that I need to re-read the instructions to my new camera in order to fully understand them.” Here, the individual's current, ongoing (object-level) experience involves comprehension (of the instructions). Monitoring processes convey information that the understanding of the instructions is, in this case, insufficient. Control processes convey information that the behavior needs to be adjusted and, in this case, how to improve comprehension (by re-reading the instructions; see Dunlosky \& Bjork, 2008, for additional summary characterizations and applications of this model). 
The Metacognitive Model has stimulated a great deal of basic and applied research on metacognition (Dunlosky \& Bjork, 2008; Perfect \& Schwartz, 2002). The model’s characterization of the relationship between object-level and meta-level experience provides a parsimonious framework for testing hypotheses concerning the capacity to monitor and regulate one’s thoughts, feelings, and behaviors (see Dimaggio \& Lysaker, 2010; Semerari et al., 2003, for recent work on the relationship between deficits in metacognition and mental illness).

\subsection{How has metacognition been assessed?}

Typically, measures of metacognition include an individual's self-report on her current experience in the context of an experimentally-controlled task. The individual might be directed to assess how well she understands a text passage as she reads it (metacomprehension), or how confident she is she will recall the important points in the passage at a later time (metamemory). Although there is general agreement that these ('concurrent probe') techniques themselves require cognitive resources, this concern is balanced by the advantage of assessing metacognition as closely as possible to its occurrence (see Dunlosky \& Bjork, 2008; Garner \& Alexander, 1989; Nelson, 1996, for extended discussions of advantages and disadvantages of different approaches to measuring metacognitive skills). Further, in studies of metacognition in the waking state, the investigator has control over the experimental conditions themselves (e.g., the difficulty of the text passage).

\subsection{Cross-state investigations of metacognition}

In recent years, powerful neuroimaging techniques such as functional magnetic resonance imaging (fMRI) and transcranial magnetic stimulation (TMS) have permitted cognitive neuroscientists to test ever-more sophisticated hypotheses concerning the relationship between subjective reports, cognitive performance, and patterns of neural activation (Baars \& Gage, 2010; 
Fuster, 2003). These rapid technological developments have created compelling opportunities to investigate the neural correlates of very specific cognitive processes, including metacognition and executive functions more generally. For example, considerable evidence has accrued highlighting the importance of fronto-cortico networks, notably prefrontal and midfrontal areas, in executive and metacognitive processing (see Fernandez-Duque et al., 2008; Fuster, 2008; Schwartz \& Bacon, 2008; Shimamura, 2008, for reviews). ${ }^{1}$

This explosion of research on the cognitive neuroscience of conscious states has magnified the importance of developing equally precise measures of subjective experience (Nielsen \& Stenstrom, 2005). As aptly noted by Allan Hobson and his colleagues:

"If the psychophysiology and neuropsychology of mental life are to advance, we must develop the means of characterizing and quantifying the subjective experience of conscious states . . . no amount of technical sophistication can compensate for neglect of exactly what psychological features the neurobiological data are asked to explain” (Hobson, Pace-Schott, \& Stickgold, 2003, p. 231).

\subsection{Why is the MACE needed?}

Valid and reliable self-report measures of high-order cognition are critical to investigations of the cognitive neuroscience of conscious states. For example, there is persistent disagreement among theorists as to whether high-order cognition in dreaming is deficient or is essentially continuous with waking cognition (see Hobson et al., 2003; Kahan, 2001; Wolman \& Kozmovà, 2007, for reviews). A precise characterization of the patterns of neural activation associated with variations in reported high-order cognition across states is challenging because current methods of metacognition in the waking state (e.g., concurrent probe techniques) cannot 
be used to investigate metacognition in sleep. Thus an instrument that targets high-order cognition and that is feasible to use across conscious states (e.g., dreaming, waking) is needed.

\subsection{The present research}

This paper presents the findings from several studies examining the psychometric properties of the Metacognitive, Affective, and Cognitive Experiences (MACE) Questionnaire, an instrument which targets the core components of metacognition - monitoring and control (Flavell, 1979; Nelson \& Narens, 1990; 1994; Son \& Schwartz, 2002). Initial evidence that the MACE is a psychometrically sound instrument that can be used to assess metacognition across states has emerged from several previous studies of higher cognition in dreaming and waking (see Kahan \& LaBerge, 2011, for a review of these studies). These studies reveal strikingly consistent patterns in responses to the MACE in comparisons of dreaming and waking experiences. The similarities and differences in MACE responses for dreaming and waking experiences were, with few exceptions, consistent across different samples (lucid dreamers, non-lucid dreamers), different response scales (discrete, continuous), and different sampling conditions (mail-in, home, sleep laboratory, first-person vs. third-person ratings (Kahan, 2001). For example, in Kahan and LaBerge (1996), 43 undergraduates provided narrative reports of one well-remembered dream and one waking experience. Participants used the MACE to rate whether particular cognitive and metacognitive processes characterized the reported experience. For each 'yes' response, the participant also described an example of that process (attention, internal commentary, selfreflection, etc) from the target dreaming or waking experience. Trained judges who were not otherwise involved in the study used the MACE to assess the incidence of the same cognitive and metacognitive processes in the participants' narrative reports. In general, a higher incidence of high-order cognition was noted in participants’ MACE assessments of their dreaming and waking 
experiences than was noted by the judges in the participants' narrative reports. Importantly, the same patterns of high-order cognition in dreaming and waking were found, whether ratings were made by the participant (first-person ratings) or by trained raters (third-person ratings). Hence, although the Kahan and LaBerge (1996) study was primarily concerned with comparing highorder cognition across dreaming and waking, their results also provide evidence of the reliability of the MACE.

\subsubsection{Summary of current method}

A formal psychometric analysis of the MACE was conducted using data from two studies of the relationship between dreaming and waking experience (Kahan et al., 2007; Kahan, Luther, \& Thompson, 2011b). In both Study $1(N=91)$ and Study $2(N=93)$, participants used the MACE to assess the incidence of high-order cognition in two waking experiences and two dreaming experiences. Experience samples were obtained following the systematic sampling procedure summarized in the Method section (see Kahan et al, 2011b, for a detailed description of the full protocol). Interitem and test-retest correlations were used to assess reliability. Factorial, convergent and discriminant validity were analyzed to determine whether the MACE, in fact, measures the monitoring and control processes that characterize metacognition. Additional information about the instrument's reliability and validity was provided by an analysis of internal consistency (i.e., the relationship between the factors and total scores; Anastasi \& Urbina, 1997, p. 129).

\subsection{Item Development}

The MACE is a 10-item questionnaire designed to measure the ability to monitor and control one's ongoing experience-high-order cognitive skills that are the core components of metacognition (Dunlosky \& Bjork, 2008; Nelson \& Narens, 1990; 1994). Whereas most measures 
of metacognition emphasize the monitoring and regulation of one's cognitive processes, the MACE assesses the monitoring and regulation of both cognitive and affective experience (see Fernandez-Duque et al., 2000, pp. 294-295, for a discussion of emotion regulation as metacognition). The version of the MACE used in the present studies is the third iteration of the instrument (see Kahan \& LaBerge, 1996; Kahan et al., 1997; Kahan \& LaBerge, 2011 for discussion of the development and pilot testing of the MACE).

The MACE includes four self-monitoring questions that assess conscious noticing: when one’s attention is captured: “Did you find your attention suddenly captured by something?”; current emotions: “Did you feel any emotions during the experience?”; internal commentary: “Did you comment to yourself about any person or event?”; and concern over the impression one made: "Were you concerned about the impression you made, how you looked or how you appeared to others?” The latter question was based on the Public Self-Consciousness subscale of the Self-Consciousness Scale (Fenigstein, Scheier, \& Buss, 1975).

The MACE also assesses self-reflective consciousness (Metcalfe, 2008) or reflective awareness, which is defined as "awareness that is focused on subjective experience - awareness of ongoing thoughts, feelings, or actions” (Kahan \& LaBerge, 1994, p. 248; see Kahan \& LaBerge, 2011, p. 495, for a comparison of these and related terms). Earlier versions of the MACE included one reflective awareness question: "Did you think about your own thoughts, feelings, attitudes, motivations, or behavior?” (Kahan et al., 1997, p. 137). This question was inspired by the Dream Self-Reflectiveness (DSR) scale, specifically Category 7, developed by Allan Moffitt and his colleagues (Purcell, Mullington, Moffitt, Hoffmann, \& Pigeau, 1986), which is based on Rossi's conceptualization of the development of self-reflection (Rossi, 1985/1972, pp. 154-161). The version of the MACE used in the present study includes three reflective awareness questions: 
monitoring of one’s internal experience: “Did you think about your own thoughts or feelings?”; monitoring of one’s behavior: “Did you think about what you were doing?”; and monitoring the external environment: “Did you think about what was happening around you?”. Three reflective awareness questions were included to assess whether the incidence of reflective awareness across states (i.e., dreaming, waking) varies with the object of reflection, notably internal experience versus external conditions (Duvall \& Wicklund, 1972).

Finally, three MACE questions target self-regulatory behaviors, the 'control' aspect of metacognition (Posner \& Rothbart, 1998; Son \& Schwartz, 2002). These questions ask the participant to assess whether she made a choice or decision: "Did you choose between two or more options?”; focused her attention on a task: “Did you focus for a period of time on accomplishing a particular task?”; or experienced unusual difficulties or thwarted intentions in a task: "Did you experience any unusual difficulty in accomplishing anything you were trying to do?”.

\section{Study 1: internal consistency, exploratory factor analysis, and factor intercorrelations}

\subsection{Method}

\subsubsection{Sample and participant selection}

Participants were 206 students enrolled in introductory or other lower division psychology courses at Santa Clara University, a private liberal arts university in Northern California. All participants were volunteers and they received either partial course credit or $\$ 25.00$ for their participation. The experimental protocol was approved by the university’s Institutional Review Board and participants were treated in accordance with APA Ethical Guidelines (APA, 1992). As part of a larger study, 452 students completed an online screening questionnaire. To be eligible for the study, participants had to: (1) report regular bedtimes and waking times (responses of 
"usually" or "always" to the questions: "In a typical week, do you go to bed within an hour of the same time every night?" "In a typical week, do you get up within an hour of the same time each morning?”) and (2) report dream recall on at least two mornings in a typical week (response of “2” or higher to the question: "During a typical week, on how many mornings do you remember at least 1 dream?”). Participants also had to deny smoking and the use of prescription medications that impact sleep or mood.

Selection criteria were met by 206 students; these individuals were invited to participate in the study. Data from 22 participants (11\%) were eliminated because of incomplete protocols or failure to follow instructions. The final group of 184 participants included 64 males (18 - 31 yrs, $M=19.97, S D=2.56)$ and 120 females $(18-27 \mathrm{yrs}, M=18.95, S D=1.40)$. This group was comprised of two samples, separated in time by roughly one year. Sample $1(N=91)$ included 36 males (18 - 28 yrs, $M=20.7, S D=2.46)$ and 55 females $(18-27$ yrs, $M=19.3, S D=1.73)$. Sample $2(N=93)$ included 28 males $(18-31$ yrs, $M=19.1, S D=2.48)$ and 65 females $(18-22$ yrs, $M=18.7, S D=.94)$. The percentage of females, $60 \%$ in Sample 1 and $70 \%$ in Sample 2, is typical of studies in dream psychology (Stepansky et al., 1998). The only difference in PIQ responses across the two samples was higher ratings on "stability of dream recall” for participants in Sample $1(M=3.20, S D=.82)$ versus Sample $2(M=2.78, S D=1.31), F(1,183)=16.51, p=$ $.011,95 \%$ CI $[.09, .74]$. All participants reported English as their primary language. Differences in the procedures employed with the two samples are described in section 5.2.

\subsubsection{Design and procedure}

Participants who fulfilled the selection criteria, described in section 3.1.1., attended an orientation session. Orientation sessions were conducted in groups of four. Here, a research assistant gave an overview of the study and provided information about the study's purpose, 
expectations of participants, protection of participants' confidentiality, and compensation for participating. Research assistants who conducted orientation sessions were blind to the specific hypotheses of the study. Students who elected to participate signed the informed consent and completed a number of individual differences measures and a general health questionnaire. Results from three of the individual differences measures are included in the psychometric analyses of convergent and discriminant validity of the MACE, reported in Study 3. After completing the questionnaires, participants received instruction in the protocol for sampling dreaming and waking experiences and separate packets of materials for the upcoming experience samples. A summary of the experience-sampling protocol is provided next; for a more detailed description of this protocol, see Kahan et al. (2011b).

In preparation for obtaining a dreaming sample, the participant set an alarm for 30-min prior to the usual waking time. The participant also reviewed the guidelines for dream reporting. These guidelines, used in previous studies (e.g., Kahan \& LaBerge, 1996; 2011), instruct participants to record, upon awakening from a dream, a detailed narrative of the previous fifteen minutes (approx) of the dreaming experience. Participants were instructed to describe events, thoughts, feelings, and sensations or reflections experienced during the dream; they were asked to leave out embellishments, explanations, and 'after-the-fact' commentary.

Upon awakening the next morning, the participant used the materials provided in a Dreaming Sample packet to record the dream experience according to these dream-reporting guidelines. After completing the narrative report, the participant inserted the narrative report in the packet. The protocol instructed the participant to again call the original dream experience to mind as clearly as possible and to use the MACE to rate the incidence of high-order cognition during the dreaming experience. For each question the participant answered 'yes,' he or she 
provided an exemplar. After completing the MACE, the participant completed a second questionnaire, the Subject Experiences Rating Scale (SERS). The SERS assesses the incidence and prevalence of sensory, affective, and cognitive qualities of the dream experience. Data from the cognition portion of the SERS are included in the validity analyses reported in Study 3.

For waking samples, the participant set an alarm for a time no sooner than two hours after waking and no later than two hours before bedtime. When the alarm sounded, the participant used the materials provided in a Waking Sample packet to record a narrative of the prior 15-min (approx) of their waking experience. The participant inserted the narrative report in the packet and then completed the MACE and SERS, with reference to the waking experience. Prior to data entry, all MACE exemplars were reviewed to confirm the participant understood the question.

Experience sampling took place during two, ideally consecutive, weeks. The order in which dreaming and waking experiences were sampled was counter-balanced across week $(1,2)$ and state (dreaming, waking). Participants were randomly assigned to a sampling order using block randomization, in order to minimize order effects.

In sum, participants reported and rated four events, two sampled from dreaming and two from waking. For each of the ten MACE questions, the number of 'yes' responses given by each participant was totaled across the dreaming and waking events. These totals (possible range 0-4) were entered into the analyses described next.

\subsection{Interitem consistency. Coefficient alpha (Cronbach, 1951) was calculated using SPSS}

(v.19.0); item-total and interitem correlations also were examined. Item-total correlations ranged from .18 (Q6) to .52 (Q9); most were above .30. The MACE was found to have adequate interitem consistency, alpha $=.68$ (Nunnally, 1978). Item numbers and content for each of the questions can be seen in Table 1, along with the results of the factor analysis described next. 
3.2. Exploratory factor analysis. The same weighted proportions described above were subjected to exploratory factor analysis using principal components analysis with varimax rotation (SPSS 19.0); this rotation method minimizes the number of variables that have high loadings on each factor. $^{3}$ The analysis yielded three factors with eigenvalues greater than 1.0. Factor loadings and questions can be seen in Table 1. Questions 1, 4, 5, and 9 loaded on one factor, named SelfRegulation. The content of these questions included choosing among options, focusing on a task, experiencing unusual difficulty in accomplishing something, and thinking about what one was doing. Questions 2, 3 and 10 loaded on a second factor, named Monitoring External Environment. The content of these questions included commenting to the self about a person or event, having one’s attention suddenly captured, and thinking about what was happening around one. Questions 6, 7, and 8 loaded on a third factor, named Monitoring Internal Experience. The content of these questions included being concerned about the impression one was making on others, feeling emotions, and thinking about one's own thoughts or feelings. Together, the three factors accounted for $52 \%$ of the variance in the measure. The items had factor loadings ranging from .46 - .79 and showed relatively small loadings on the remaining two factors (.38 or less).

3.3. Internal consistency. We expected weak to moderate, positive and significant correlations among the MACE factors. All factors were expected to assess the general construct of metacognition, but each was considered a measure of separable facets of metacognition (Nelson \& Narens, 1990; 1994; Son \& Schwartz, 2002). Pearson Product-Moment correlation analysis (one-tailed, $d f=183$ ) revealed that all three correlations fit the expected pattern. The correlation between Self-Regulation and Monitoring External Environment was .27, $p<.001$; the correlation between Self-Regulation and Monitoring Internal Experience was .30, $p<.001$; the correlation between Monitoring External Environment and Monitoring Internal Experience was .32, $p<$ 
.001. Per the guidelines outlined by Anastasi and Urbina (1997, p. 129), we assessed the criterion of internal consistency by computing the correlations (Pearson's $r$ ) between the MACE factors and the MACE total score (proportion of 'yes' responses for all ten MACE questions). High correlations were observed between the three factors (Self-Regulation, Monitoring External Environment, and Monitoring Internal Experience) and the MACE total score; correlation coefficients $(d f=183)$ were, respectively, .76, .73, and .67, all $p s<.001$.

\subsection{Summary}

The MACE appeared to be internally consistent in terms of items, factor scores and total scores. Inter-item correlations were significant and the basic factor structure of the MACE was consistent with the Metacognitive Model (Nelson, 1990). Finally, the MACE factors were each strongly correlated with total MACE scores and weakly correlated with the other two factors. Additional large-scale studies will be important to confirm the stability of the factor structure within and across different experiential conditions.

\section{Study 2: test-retest reliability}

\subsection{Participants}

Participants in Sample $2(N=93)$ of Study 1 contributed data for the assessment of test-retest reliability.

\subsection{Procedure}

Participants in Sample 2 followed the same sampling protocol as described in section 3.1.2, with two variations. As was the case with Sample 1, Sample 2 participants completed the MACE, inserted it in an envelope, seal it, and completed the Subjective Experiences Rating Scale (SERS), and Profile of Mood States (POMS). Unlike Sample 1 participants, Sample 2 participants then completed the MACE (and SERS) a second time. Instructions were to (again) 
call to mind their experiences right before they were signaled. Participants were instructed to respond to the MACE questions with respect to the original experience and not try to recall what they had reported on their first MACE. Participants put the second MACE in a separate envelope, sealed it, and then completed the SERS a second time. In the latter case, the time interval between the first and second application of the MACE was approximately 10 minutes. This delay was long enough to reduce possible recency and priming effects that could influence MACE judgments had participants completed the second application of the MACE immediately after the first. At the same time, this delay was short enough to mitigate the influence of episodic memory processes that can influence the reconstruction of recent experience (e.g., Hurlburt \& Heavey, 2008).

As described earlier, each participant assessed (and re-assessed) four events. These four events represented experience-sampling across two weeks and across two states (dreaming, waking).

\section{Study 2: results}

Test-retest reliability was computed for 372 events (93 participants x 4 events each). For each of the four events (two dreaming, two waking), total scores were calculated by adding the number of “yes” responses on the ten MACE questions. Total scores for the second application of the MACE were computed in a similar fashion, again for each of the four events. Correlations between the first and second assessment for the four events were: Dream 1 (.92), Dream 2 (.81), Wake 1 (.87), Wake 2 (.97). The average test-retest correlation between total MACE scores for the first and second assessment was .90. All inter-correlations were significant at $p<.001$. Testretest reliability also was computed for each of the three factors, again for each of the four events. Average Pearson Product-Moment correlations for the three factors were: .86, .89, and .82 for Self-Reg, MEE, and MIE factors, respectively. Scores for the three factors were calculated with 
all events included. Overall reliability coefficients for the three factors, calculated from all 372 events were: .93 (Self-Regulation), .95 (Monitoring External Environment), and .88 (Monitoring Internal Experience).

In sum, correlations between the first and second assessments revealed strong test-retest reliability across different events (first, second), different states (dreaming, waking), and for all three MACE factors.

\section{Study 3: relationships between the MACE and related constructs}

7.1. Participants. Participants from both samples contributed data to these analyses.

7.2. Measures and predictions. Three individual differences measures completed during the orientation session were used to assess convergent and discriminant validity: the Kentucky Inventory of Mindfulness Skills Scale (KIMS; Baer, Smith, \& Allen, 2004), the SelfConsciousness Scales (SCS; Fenigstein et al., 1975), and the Experiences Questionnaire (EQ; Fresco et al., 2007). Only participants in Sample 2 completed the EQ. A measure of the cognitive qualities of dreaming and waking experiences, obtained from the Subjective Experiences Rating Scale (SERS), also was included.

\subsubsection{Individual differences measures.}

Results of the individual differences measures relevant to the present psychometric analysis are discussed in detail below. Results of the other measures and comparisons of dreaming and waking are presented in other papers (Kahan, Bulkeley, \& Kawadri, 2011a; Kahan et al., 2011b).

7.2.1.1. All participants completed the Kentucky Inventory of Mindfulness Skills (KIMS), a 39item scale developed by (Baer et al., 2004) to assess "the general tendency to be mindful in daily life” (p. 193). Baer et al. developed their measure based on the definition of mindfulness offered 
by Kabat-Zinn (1994) and others: “focusing one’s attention in a nonjudgmental or accepting way on the experience occurring in the present moment” (p. 191). The KIMS assesses four mindfulness skills (Baer et al., pp. 193-194): Observing: noticing internal (thoughts, feelings, sensations) or external phenomena (sounds, smells); Describing: verbal descriptions or labeling of ongoing experience, without judging or analyzing the experience; Acting with Awareness: focusing one’s full awareness on one thing at a time; Accepting without Judgment: nonjudgmental acceptance or allowing of current experience; the individual refrains from "applying evaluative labels such as good/bad” (p. 194).

The psychometric properties of the KIMS were analyzed and reported by Baer et al (2004). In two student samples ( $N$ s $=205$, 215, respectively), the KIMS showed a clear fourfactor structure consistent with the mindfulness skills described above. The measure also showed "high internal consistency and adequate to good test-retest reliability" (p. 203). ${ }^{4}$

\subsubsection{Of 184 total participants, 175 (95\%) completed the Self-Consciousness Scale (SCS;}

Fenigstein et al., 1975). The SCS is a twenty-three item scale used to assess individual differences in self-consciousness: "the consistent tendency of persons to direct attention inward or outward" (Fenigstein et al., p. 522). This construct emphasizes self-focused attention, which involves selfawareness (awareness of thoughts, feelings, behavior, or appearance) self-examination (selfmonitoring; reflecting, fantasizing or daydreaming about the self), and intentionality (making decisions or plans that involve the self. Principal components factor analysis (varimax rotation) determined that the SCS is comprised of three factors: Private Self-Consciousness (PrSC), Public Self-Consciousness (PublSC), and Social Anxiety (SocAnx). According to Fenigstein et al., there is a qualitative difference between the two SC subscales and social anxiety: "Public and private SC refer to a process of self-focused attention; social anxiety refers to a reaction to this process” 
(p. 523; emphasis in original paper). In addition to the clear three-factor structure, the SCS showed good test-retest reliability. The SCS has been used extensively in clinical, cognitive, personality, and self-perception research to assess self-consciousness (see Fenigstein, 2009, for a review). The SCS, both in its original (Fenigstein et al., 1975) and revised form (Burkrant \& Page, 1984), has shown robust psychometric properties and, with few exceptions, strong cultural validity (Wu \& Watkins, 2006). ${ }^{5}$

\subsubsection{Experiences Questionnaire (EQ)}

Of the 93 participants in Sample 2, eighty-five (91\%) completed an additional individual difference measure — the Experiences Questionnaire (EQ), originally developed by Teasdale and his colleagues as a measure that would be sensitive to changes in cognitive style during the course of psychotherapy, especially cognitive-behavior therapy (CBT; e.g., Teasdale et al., 2002). In particular, the EQ was designed to measure of decentering: “the ability to observe one’s thoughts and feelings as temporary, objective events in the mind” (Fresco, Segal, Buis, \& Kennedy, 2007, p. 447). Psychometric analyses of the EQ conducted by Fresco and his colleagues confirmed the EQ as a single-factor scale with good convergent and discriminant validity, as well as strong testretest reliability (Fresco et al., 2007).

\subsubsection{Measure of phenomenal characteristics of subjective experience}

The Subjective Experiences Rating Scale (SERS) was developed by the first author, based on the Memory Characteristics Questionnaire (MCQ) developed by Marcia Johnson and her colleagues to assess the incidence of particular phenomenal qualities of subjective experience (e.g., Johnson, Foley, Suengas, \& Raye, 1988; Johnson, Kahan, \& Raye, 1984; Suengas \& Johnson, 1988). Participants use a Likert-type scale (0 'not at all' to 5 'a lot') to rate the extent to which their subjective experience includes various sensory, affective, and cognitive qualities. This 
measurement approach has been used in numerous studies of the phenomenal qualities of autobiographical memories (e.g., Mather, Henkel, \& Johnson, 1997; Schaefer \& Philipott, 2005).

\subsection{Summary of predictions}

The KIMS, SCS, and EQ were selected as comparison measures because the constructs of mindfulness, decentering, self-consciousness, and metacognition all emphasize the capacity for noticing or monitoring one's current subjective experience (self-focused attention). Examining the relationships in this nomological net allowed us to assess the convergent and discriminant validity of the MACE as a measure of metacognition (i.e., as related to, but distinct from, these constructs). Based on the shared emphasis on self-focused attention, we expected to find weak, positive correlations among the subscales and factors that focus on monitoring one's subjective experiences. Specifically we expected to find that the MACE factors Self-Regulation and Monitoring External Environment would be related to the SCS subscales Public and Private SelfConsciousness. In contrast, based on the distinctiveness of the constructs assessed by each measure, we expected to find higher intercorrelations among subscales within each measure compared to the intercorrelations of subscales between measures. For the MACE specifically, we expected to find no significant correlations between MACE total scores and total scores on the comparison measures, because the MACE is intended to discriminately measure the incidence of core metacognitive skills (monitoring and control) associated with recollected, recent experience and, unlike the other measures, has a strong emphasis on goal-directed behavior. Moreover, we expected to find significant correlations between the EQ and the KIMS (especially the Acceptwithout-Judgement subscale), because both constructs emphasize a general disposition of nonjudgmental acceptance of present-moment experience, i.e., monitoring without control). 
The SERS was selected as a comparison measure to further establish discriminant validity. The SERS assesses a range of cognitive events that, for the most part, do not necessitate the selfobservation, intentionality, or self-regulation inherent in metacognition. We predict that there will be no significant correlations between the total scores of the two measures and that the intercorrelations between the SERS and MACE factors will be weaker than the intercorrelations among cognitive events (SERS) or among MACE factors. We do expect some weak relationships among MACE factors and specific cognitive events (e.g., thinking, evaluating); these specific predictions are elucidated in Section 8.2.4.

\section{Study 3: results and discussion}

\subsection{Relationships between MACE subscales and other constructs.}

Pearson's $r$ was used to compute correlations among the MACE and the three individual differences measures. Table 2 presents the intercorrelations among the MACE, the SERS cognitive subscale, and the three individual difference measures, the KIMS, SCS, and EQ, designed to assess, respectively, mindfulness, self-consciousness, and decentering.

8.1.1. Mindfulness. As expected, total scores for the MACE and KIMS were not related $(r=.02$; $p=.83$ ) and the intercorrelations among the KIMS subscales (which were consistent with past research, see Baer et al., 2004, Table 2, p. 197) were stronger than the correlations between the KIMS subscales and the MACE. Only one of twelve correlations between the KIMS and MACE subscales was significant. The KIMS Describe subscale was significantly, but weakly, related to Monitoring Internal Experience $(r=.17, p<.05)$.

This relationship, though not predicted, makes sense given that the describing one's experiences in words is a natural feature of monitoring one's current internal experiences (also see the discussion in Baer et al., 2004, p. 193); consistent with this view-KIMS Describe scores 
are positively correlated with participants’ PrivSC scores but negatively correlated with their PublSC and SocAnx scores (Table 2).

8.1.2. Self-Consciousness. As expected, total scores for the MACE and the SC scale were not related $(r=.09 ; p=.26)$. The pattern of intercorrelations among the SC subscales replicated past research: PublSC was related to both PrivSC and SocAnx; PrivSC was not related to SocAnx (Fenigstein et al., 1975, p. 525). As seen in Table 2, correlations among SC subscales were stronger than those between the MACE subscales and the SC subscales. Consistent with predictions, three of the nine correlations among SC subscales and MACE subscales were significant. The PrivSC subscale was significantly, but weakly, related to MACE Self-Regulation and Monitoring Internal Experience ( $r s=.17, .16$, respectively; $p s<.05)$. The PublSC subscale was significantly, but weakly, related to Monitoring Internal Experience $(r=.14, p<.05)$.

8.2.3. Decentering. As expected, decentering scores were not strongly related to the MACE. Surprisingly, decentering was negatively related to one MACE component, Monitor Internal Experience, $r=-.21, p=.03$. This result is consistent with the claim that metacognitive monitoring occurs within an evaluative, goal-directed context whereas decentering does not. As expected, decentering scores were positively correlated with three of the four KIMS subscales. The strongest convergence was between the KIMS subscale “Accept without Judgment” and Decentering, $r=.31, p<.01$.

8.2.4. Cognition. As predicted, the total MACE score was not related to the total score for SERS Cognition measure $(r=-.08 ; p=.49)$. Of the three MACE subscales, Self-Regulation was positively, but weakly, related to SERS Cognition $(r=.19, p<.05)$. This relationship makes sense when we consider the relationship between MACE factors and individual SERS items (Table 3). Although total scores for the MACE and SERS Cognition were, as anticipated, 
unrelated, some items on the SERS were expected to share variance with the MACE. Specifically, because thinking is inherent to metacognition, we expected all three MACE factors to be correlated with SERS ratings of "thinking.” Because, self-regulation, by definition, includes not only thinking, but also planning, evaluating, and attending to one’s inner experience, we expected that the MACE Self-Regulation subscale would be correlated with these SERS items. Following similar logic, the MACE Monitoring Internal Experience should be related to SERS ratings of “thinking” and "attending to inner world.” and the MACE Monitoring the External Environment factor should be related to SERS ratings of "thinking” and "attending outward." As seen in Table 3, these hypotheses were confirmed in the pattern of intercorrelations between the MACE factors and SERS ratings.

\subsection{Summary}

The pattern of intercorrelations among the MACE, KIMS, and the Decentering scale is consistent with the assertion that the measures of mindfulness and decentering should be more strongly related to each other than to a measure of metacognition. The MACE shows good discriminant validity in that MACE scores were not strongly related to the two measures that emphasize non-judgmental self-monitoring (i.e., the KIMS and EQ).

Convergent validity is seen in the weak, yet significant, relationships between the MACE and the SERS cognition subscale and between the MACE and the Private Self-Consciousness subscale of the SCS, these being the two subscales that tap constructs most closely related to monitoring and control. The construct validity of the MACE is further reinforced by the strong correlations among the MACE factors in contrast to the weak relationship between the MACE and the other measures.

\section{General discussion}


The intent of this research was to analyze the psychometric properties of a self-report measure of metacognition, the Metacognitive, Affective, Cognitive Experience (MACE) Questionnaire. The psychometric evidence indicates that the MACE is a reliable and valid measure of metacognition across waking and dreaming.

Previous research indicated that the MACE had strong inter-rater reliability (Kahan \& LaBerge, 1996). The current study provides additional evidence of the reliability of the measure, including consistency among responses to individual items, consistency among scores on the MACE factors, and consistency among total scores on separate administrations given over time (i.e., test-retest reliability). This consistency emerged using samples from dreaming and waking experiences.

Factorial, convergent, and discriminant validity were used to explore whether the MACE measures metacognition. The emergence of factors consistent with a theoretical understanding of metacognition (i.e., that metacognition involves monitoring and control) provide evidence for the factorial validity of the measure. Interestingly, the factor structure includes one control component and two monitoring components, one that emphasizes monitoring internal experience (thoughts, feelings) and one that emphasizes monitoring external conditions. The emergence of two monitoring factors is consistent with the theorizing of Duvall and Wicklund (1972), with Fenigstein et al's conceptualization of private and public self-consciousness (Fenigstein et al., 1975; Fenigstein, 2009), and with recent research in cognitive neuroscience demonstrating that different fronto-cortico areas are activated when self-reflection is more inward-directed versus outer-directed (Johnson et al., 2006).

To evaluate convergent and discriminant validity, metacognition was conceptualized as embedded in a nomological net that included focused attention/self-monitoring, mindfulness, 
decentering, self-consciousness, and cognition. The pattern of relationships among the MACE and measures assessing one or more of these constructs provided evidence that the MACE measures self-focused attention and goal-directed behavior; moreover, the evidence suggests that the MACE is clearly distinct from the related concepts of cognition, mindfulness, selfconsciousness, and decentering. Taken together, these results indicate that the MACE is a valid measure of metacognition.

\subsection{Limitations}

The reliance on student samples may be considered a general limitation of the present studies, insofar as external validity is restricted. Regarding the cultural validity of the MACE, it is possible that the factor structure could be different among practitioners of Buddhism or Hinduism wherein self-focused attention is less heavily emphasized, dreaming and waking are viewed as part of a seamless continuum of consciousness, and dreaming is as often seen as a collective, spiritual experience as it is a personal one (see, especially, Bulkeley, 2008; Hurd, 2011).

The intent in developing the MACE was to construct a measure that could be used to assess metacognition in both dreaming and waking experience (Kahan \& LaBerge, 1996; Kahan et al., 1997). Thus, studies of the content and process features of dreaming require the participation of individuals with reliable dream recall. Participants in the present studies were moderate to high dream-recallers, according to Schredl and Reinhart's (2008) criteria. This selection bias, necessary as it is to the study of cross-state cognition and phenomenology, may restrict the generalizability of the present findings. For example, it is possible that people with higher dream recall are generally more interested in their inner lives or more self-reflective than individuals who tend to be more externally-oriented (e.g., Hartmann, Elkin, \& Garg, 1991; Kozmovà \& Wolman, 2006). 
Another potential limitation is that training with the questionnaire may introduce possible demand characteristics by calling the participants' attention to particular qualities of subjective experience. Simply attending to one's dream experiences can increase dream recall (e.g., Schredl, Nürnberg, \& Weiler, 1996) and reflective awareness in dreaming can be increased via waking attentional practices (Price \& Cohen, 2006; Purcell et al., 1986). The simple fact of asking questions about specific qualities of subjective experience may increase the reported incidence of these qualities. In the present study, however, these concerns are lessened because the same individuals applied the MACE to both their dreaming and waking experiences. Thus, response biases or other practice effects should fall more or less equally on ratings of dreaming and waking

experiences. [ In spite of the above-mentioned issues, a strong case has been made for involving trained self-observers in studies of subjective experience (Hobson et al., 2003;Hurlburt \& Heavey, erased or a closing paren needs to be added

2008; Nielsen \& Stenstrom, 2005; Varela, Thompson, \& Rosch, 1991). A future study could incorporate the MACE in a study of techniques to increase mindfulness, DRF, or lucid dreaming and see whether there are corresponding changes in the incidence of metacognition in both waking and dreaming.

\subsection{Future directions}

Although the MACE was initially developed to index metacognitive skills in dreaming and waking, its utility is not restricted to this comparison. The MACE could easily be adapted for use in studies of HOC associated with hypnagogic imagery, hypnotically-induced states, waking fantasy, or virtual reality experiences. For example, the MACE was recently used by Gackenbach and Hunt (2011) to compare metacognition in the dreams of expert and novice gamers. The MACE also could be employed by clinicians in studies of rumination (e.g., Papageorgiu \& Wells, 2003) or of change in metacognition associated with psychotherapy (e.g., Semerari et al., 2003). 
The MACE also would be useful to social-cognitive theorists interested in the interplay of motivation, emotion, and cognition (e.g., Scholer \& Higgins, 2010) and to cognitive neuroscientists exploring the neural correlates of metacognitive skills (e.g., Johnson et al., 2006; Shimamura, 2008). An empirical question for future studies is whether the factor structure of the MACE described in the present paper, where metacognition was sampled from waking and sleep, shifts when the MACE is used to under other experiential conditions such as hypnosis, meditation, virtual reality, daytime imagery, hypnagogic or hypnopompic states, or even some drug-states.

A minimum level of cognitive expertise (and training) is needed in order to use the MACE, restricting its use with people with significant cognitive impairments and with children. It is possible, however, that the MACE could be adapted for use in developmental studies of the

emergence of high-order cognitive skills (e.g., Terrace \& Metcalfe, 2005). The MACE could also be utilized by a clinical interviewer, perhaps in conjunction with clinical measures of metacognition, such as the Metacognition Assessment Scale (Semerari et al., 2003).

\subsection{Conclusions}

The MACE was designed to assess the core processes involved in metacognition: monitoring and control, as conceptualized in the Metacognitive Model. Previous research established the MACE as a useful tool for comparing high-order cognition sampled from dreaming and waking. The three studies reported here provide further evidence of the reliability of the MACE and establish its validity as a tool for measuring metacognition sampled under varied experiential conditions. 


\section{Acknowledgements}

This research was supported in part by grants from Santa Clara University and the

Montgomery Foundation awarded to Tracey L. Kahan. A preliminary psychometric analysis of the MACE based on Study $1(N=93)$ was presented at the annual conference of the International Association for the Study of Dreams (Kahan \& Sullivan, 2007).

Special thanks to the Santa Clara University students who assisted in data collection: Megan Thompson, Emily Luther, Rebecca Gurule, Danica Zold, Patrick Rugo, Lindsey Hanneman, and Benjamin Nimmons. 
References

American Psychological Association (1992). Ethical principles of psychologists and code of conduct. American Psychologist, 57(12), 1060-1073.

doi: 10.1037/0003-066X.57.12.1060

Anastasi, A., \& Urbina, S. (1997). Psychological testing ( $7^{\text {th }}$ ed). New Jersey: Prentice Hall.

Anderson, E. M., Bohon, L. M., \& Berrigan, L. P. (1996). Factor structure of the Private SelfConsciousness Scale. Journal of Personality Assessment, 66, 144-152.

doi: 10.1207/s15327752jpa6601_11

Ardila, A. (2008). On the evolutionary origins of executive functions. Brain and Cognition, 68(1), 92-99.

doi: 10.1016/j.bandc.2008.03.003

Baars, B. (1988). A cognitive theory of consciousness. Cambridge: Cambridge University Press.

Baars, B. J., \& Gage, N. M. (2010). Cognition, brain, and consciousness: An introduction to cognitive neuroscience ( $2^{\text {nd }}$ ed.). Academic Press.

Baer, R. A., Smith, G. T., \& Allen, K. B. (2004). Assessment of mindfulness by self-report: The Kentucky Inventory of Mindfulness Skills. Assessment, 11(3), 191-206. doi: 10.1177/1073191104268029

Brown, A. L., Campione, J. C., \& Day, J. D. (1981). Learning to learn: On training students to learn from text. Educational Researcher, 10, 14-21.

Bulkeley, K. (2008). Dreaming in world religions: A comparative history. New York: NYU Press.

Burnkrant, R. F., \& Page, T. J. Jr. (1984). A modification of the Fenigstein, Scheier, and Buss Self-Consciousness Scales. Journal of Personality Assessment, 48, 629-637. 
doi: 10.1207/s15327752jpa4806_10

Cronbach, J. L. (1951). Coefficient alpha and the internal structure of tests. Psychometrika, 16, 297-334.

Dimaggio, G., \& Lysaker, P. H. (Eds.)(2010). Metacognition and severe adult mental disorders: From research to treatment. New York: Routledge/Taylor \& Francis Group.

Domhoff, G. W. (2003). The scientific study of dreams: Neural networks, cognitive development, and content analysis. Washington, DC: American Psychological Association. doi: $10.1037 / 10463-000$

Dunlosky, J., \& Bjork, R. A. (2008). Handbook of metamemory memory. New York: Psychology Press.

Duvall, S., \& Wicklund, R. A. (1972). A theory of objective self-awareness. New York: Academic Press.

Fenigstein, A., Scheier, M. F., \& Buss, A. H. (1975). Public and private self-consciousness: Assessment and theory. Journal of Clinical and Consulting Psychology, 522-527. doi: 10.1037/h0076760

Fenigstein, A. (2009). Private and public self-consciousness. In M. R. Leary \& R. H. Hoyle (Eds.), Handbook of individual differences in social behavior. New York, NY: Guildford Press.

Fernandez-Duque, D., Baird, J. A., \& Posner, M. I. (2000). Executive attention and metacognitive regulation. Consciousness and Cognition, 9, 288-307.

Flavell, J. H. (1979). Metacognition and cognitive monitoring: A new area of cognitive-developmental inquiry. American Psychologist, 34, 906-911. 
Flavell, J. H. (1999). Cognitive development: Children’s knowledge about the mind. Annual Review of Psychology, 50, 21-45.

Flavell, J. H. (2000). Development of children’s knowledge about the mental world. International Journal of Behavioral Development, 24, 15-23.

Fresco, D. M., Moore, M. T., van Dulmen, M., Segal, Z. V., Teasdale, J. D., Ma, H., \& Williams, J. M. G. (2007). Initial psychometric properties of the Experiences Questionnaire: Validation of a self-report measure of decentering. Behavior Therapy, 38(3), 234-246. doi: 10.1016/j.beth.2006.08.003

Fresco, D. M., Segal, Z. V., Buis, T., \& Kennedy, S. (2007). Relationship of posttreatment decentering and cognitive reactivity to relapse in major depressive disorder. Journal of Consulting and Clinical Psychology, 75(3), 447-455. doi: 10.1037/0022-006X.75.3.447

Fuster, J. M. (2003). Cortex and mind: Unifying cognition. New York: Oxford University Press.

Gackenbach, J.I. \& Hunt, H. (2011). A deeper inquiry into the association between lucid dreams and video game play. In K. Bulkeley \& R. Hurd (Eds.), Lucid Dreaming Cultures. Manuscript in preparation.

Garner, R., \& Alexander, P. A. (1989). Metacognition: Answered and unanswered questions. Educational Psychologist, 24(2), 143-158. doi: 10.1207/s15326985ep2402_2

Hartmann, E. (2010). Meteorite or gemstone? Dreaming as one end of a continuum of functioning: Implications for research and for the use of dreams in therapy and self-knowledge. Dreaming, 20(3), 149-168. 
Hartmann, E., Elkin, R., \& Garg, M. (1991). Personality and dreaming: The dreams of people with very thick and very thin boundaries. Dreaming, 1, 311-324.

Heavey, C. L., \& Hurlburt, R. T., (2008). The phenomena of inner experience. Consciousness and Cognition, 17, 798-810.

doi: 10.1016/j.concog.2007.12.006

Heckhausen, J., \& Silberman, I. (1998). Motivation and self-regulation across the life span. New York, NY: Cambridge University Press.

Hobson, J. A., Pace-Schott, E.F., \& Stickgold, R. (2003). Dream science 2000: A response to commentaries on: Dreaming and the brain. In E. F. Pace-Schott, M. Solms, M. Blagrove, \& S. Harnad (Eds.), Sleep and dreaming: Scientific advances and reconsiderations (pp. 1-50). New York: Cambridge University Press.

Horton, C. L., Conway, M. A., \& Cohen, G. (2007). Memory for thoughts and dreams. In G. Cohen and M. A. Conway (Eds.). Memory in the real world (2nd ed). Psychology Press.

Hurd, R. (2011). Integral archaeology: Process methodologies for exploring prehistoric rock art on Ometepe Island, Nicaragua. Anthropology of Consciousness, 22(1), 72-94.

Johnson, M. K. (1992). MEM: Mechanisms of recollection. Journal of Cognitive Neuroscience, 4(3), 268-280.

doi: 10.1162/jocn.1992.4.3.268

Johnson, M. K., Foley, M. A., Suengas, A. G., \& Raye, C. L. (1988). Phenomenal characteristics of memories for perceived and imagined autobiographical events. Journal of Experimental Psychology: General, 117, 371-376. 
Johnson, M. K., Hashtroudi, S., \& Lindsay, D. S. (1993). Source monitoring. Psychological Bulletin, $114,3-28$.

doi: 10.1037/0033-2909.114.1.3

Johnson, M. K., Kahan, T. L., \& Raye, C. L. (1984). Dreams and reality monitoring. Journal of Experimental Psychology: General, 113(3), 329-344.

doi: 10.1037/0096-3445.113.3.329

Johnson, M. K., Raye, C. L., Mitchell, K. J., Touryan, S. R., Greene, E. J., \& Nolen-Hoeksema, S. (2006). Dissociating medial frontal and posterior cingulated activity during selfreflection. SCAN, 1, 56-64.

doi: 10.1093/scan/nsl004

Kabat-Zinn, J. (1994). Wherever you go, there you are: Mindfulness meditation in everyday life. New York: Hyperion.

Kahan, T. L. (1994). Measuring dream self-reflectiveness: A comparison of two approaches. Dreaming, 4(3), 329-344.

Kahan, T. L. (2001). Consciousness in dreaming: A metacognitive approach. In K. Bulkeley (Ed.), Dreams: A reader on the religious, cultural, and psychological dimensions of dreaming (pp. 333-360). New York: Palgrave.

Kahan, T. L., Bulkeley, K., \& Kawadri, N. (2011a). Intensity of negative emotion predicts reflective awareness in dreaming, but not in waking. Manuscript in preparation.

Kahan, T. L., \& Horton, C. L. (2011). Methodological challenges in dream science. In D. Barrett and P. McNamara (Eds.), Encyclopedia of Sleep and Dreams. New York: ABC-CLIO.

Kahan, T. L., \& LaBerge, S. (1994). Lucid dreaming as metacognition: Implications for cognitive science. Consciousness and Cognition, 3, 246-264. 
doi: 10.1006/ccog.1994.1014

Kahan, T. L., \& LaBerge, S. (1996). Cognition and metacognition in dreaming and waking: Comparisons of first and third-person ratings. Dreaming, 6(4), 235-247.

Kahan, T. L., \& LaBerge, S. (2011). Dreaming and waking: Similarities and differences revisited. Consciousness and Cognition, 20, 494-514.

doi: 10.1016/j.concog.2010.09.002

Kahan, T. L., \& LaBerge, S., Levitan, L., \& Zimbardo, P. (1997). Similarities and differences between dreaming and waking cognition: An exploratory study. Consciousness and Cognition, 6, 132-147.

doi: 10.1006/ccog.1996.0274

Kahan, T. L., Luther, E., \& Thompson, M. (2011b). Individual differences as predictors of metacognition in dreaming and waking. Manuscript in preparation.

Kahan, T. L., \& Sullivan, K. (2007). Assessing metacognitive skills in waking and sleep: A psychometric analysis of the metacognitive, affective, cognitive experience (MACE) questionnaire. Paper presented at the annual conference of the IASD, June, Sonoma State University.

Kontogiorge, G., \& Horton, C.L. (2010). Metacognition in sleep and wake: The role of selfreflection and self-regulation in the continuity of consciousness. Manuscript in preparation.

Kozmovà, M., \& Wolman, R. N. (2006). Self-awareness in dreaming. Dreaming, 16, 196-214. Leonesio (2008). Feeling-of-knowing accuracy and recollective experience. In J. Dunlosky, \& R. A. Bjork (Ed.). Handbook of metamemory and memory (pp. 195-210). New York: Psychology Press. 
Mather, M., Henkel, L., \& Johnson, M. K. (1997). Evaluating characteristics of false memories: Remember/know judgments and memory characteristics questionnaire compared. Memory \& Cognition, 25, 826-837.

Mazzoni, G., \& Nelson, T. O. (1998)(Eds.). Metacognition and cognitive neuropsychology: Monitoring and control processes. Mahwah, NJ: Lawrence-Erlbaum Associates.

Metcalfe (2008). Evolution of metacognition. In J. Dunlosky, \& R. A. Bjork (Eds.). Handbook of metamemory and memory (pp. 29-46). New York: Psychology Press.

Metcalfe, J., \& Shimamura, A. (1994). Knowing about knowing. MIT Press.

Nelson, T. O. (1996). Consciousness and metacognition. American Psychologist, 51, 102-116. doi: 10.1037/0003-066X.51.2.102

Nelson, T. O. (2000). Consciousness, self-consciousness, and metacognition. Consciousness and Cognition, 9, 220-223.

Nelson, T. O., \& Narens, L. (1990). Metamemory: Theoretical framework and new findings. In G. H. Bower (Ed.), The Psychology of Learning and Motivation (Vol. 26, pp. 125-141). San Diego, CA: Academic Press.

Nelson, T. O., \& Narens, L. (1994). Why investigate metacognition? In J. Metcalfe and A. P. Shimamura (Eds.), Metacognition: Knowing about knowing (pp. 1-11). Cambridge, MA: Bradford Books.

Nielson, T. A., \& Stenstrom, P. (2005). What are the memory sources of dreaming? Nature, 437(27), 1286-1289.

doi: 10.1038/nature04288

Nunnally, J. C. (1978). Psychometric theory (2nd ed.). New York: McGraw-Hill. 
Nystedt, L., \& Ljungberg, A. (2002). Facets of private and public self-consciousness: Construct and discriminant validity. European Journal of Personality, 16, 143-159.

doi: 10.1002/per.440

Papageorgiu, C., \& Wells, A. (2003). An empirical test of a clinical metacognitive model of rumination and depression. Cognitive Therapy and Research, 27(3), 261-273.

Paulsson, T., \& Parker, A. (2006). The effects of a two-week reflection-intention training program on lucid dream recall. Dreaming, 16(1), 22-35.

doi: 10.1037/1053-0797.16.1.22

Perfect, T. J., \& Schwartz, B. L. (Eds.)(2002). Applied metacognition. Cambridge University Press, New York, NY.

\section{doi: 10.1017/CBO9780511489976}

Posner, M. I., \& Rothbart, M. K. (1998). Attention, self-regulation and consciousness. Philosophical Transactions of the Royal Society of London, 353, 1915-1927.

Purcell, S., Moffitt, A., \& Hoffmann, R. (1993). Waking, dreaming, and self-regulation. In A. Moffitt, M. Kramer, R. Hoffmann (Eds), The functions of dreaming (pp. 197-260). Albany: State University of New York Press.

Purcell, S., Mullington, J., Moffitt, A., Hoffmann, R., \& Pigeau, R. (1986). Dream self-reflectiveness as a learned cognitive skill. Sleep, 9, 423-437.

Rossi, E. L. (1972/1986). Dreams and the growth of personality. New York: Pergamon.

Schaefer, A., \& Philippot, P. (2005). Selective effects of emotion on the phenomenal characteristics of autobiographical memories. Memory, 13(2), 148-160.

Scholer, A. A., \& Higgins, E. T. (2010). Regulatory focus in a demanding world. In Handbook of personality and self-regulation (pp. 291-314). Wiley-Blackwell. 
Schredl, M., \& Hofmann, F. (2003). Continuity between waking activities and dream activities. Consciousness and Cognition, 12, 298-308.

doi: 10.1016/S1053-8100(02)00072-7

Schredl, M., \& Reinhard, I. (2008). Gender differences in dream recall: A meta-analysis. Journal of Sleep Research, 17, 125-131.

doi: 10.1111/j.1365-2869.2008.00626.x

Schneider, W., \& Lockl, K. (2008). Procedural metacognition in children: Evidence for developmental trends. In J. Dunlosky, \& R. A. Bjork (Eds.), Handbook of metamemory and memory (pp. 391-409). New York: Psychology Press.

Schredl, M. (2003). Continuity between waking and dreaming: A proposal for a mathematical model. Sleep and Hypnosis, 5(1), 38-52.

Schwartz, B. L., \& Bacon, E. (2008). Metacognitive neuroscience. In J. Dunlosky, \& R. A. Bjork (Eds.), Handbook of metamemory and memory (pp. 355-372). New York: Psychology Press.

Semerari, A., Carcione, A., Dimaggio, G., Falcone, M., Nicolo, G., Procacci, M., \& Alleva, G. (2003). How to evaluate metacognitive function in psychotherapy? The Metacognition Assessment Scale and its applications. Clinical Psychology and Psychotherapy, 10, 238261.

doi: 10.1002/cpp.362

Shapiro, S., \& Carlson, L. E. (2009). The art and science of mindfulness: Integrating mindfulness into psychology and the helping professions. Washington, DC: American Psychological Association.

Shimamura, A. P. (2008). A neurocognitive approach to metacognitive monitoring and control. 
In J. Dunlosky, \& R. A. Bjork (Eds.), Handbook of metamemory and memory (pp. 373390). New York: Psychology Press.

Son, L. K., \& Schwartz, B. L. (2002). The relation between metacognitive monitoring and control. In T. Perfect and B. Schwartz (Eds.). Applied Metacognition (pp. 15-38). Cambridge: Cambridge University Press.

doi: 10.1017/CBO9780511489976.003

Stepansky, R., Holzinger, B., Schmeiser-Rieder, A., Saletu, B., Junze, M., \& Zeitlhofer, J. (1998). Austrian dream behavior: Results of a representative population survey.

Dreaming, 8, 23-30.

doi: 10.1023/B:DREM.0000005912.77493.d6

Suengas, A. G., \& Johnnson, M. K. (1988). Qualitative effects of rehearsal on memories for perceived and imagined complex events. Journal of Experimental Psychology: General, 117, 377-389. doi: 10.1037/0096-3445.117.4.377

Teasdale, J. D., Moore, R. G., Hayhurst, H., Pope, M., Williams, S., \& Segal, Z. V. (2002). Metacognitive awareness and prevention of relapse in depression: Empirical evidence. Journal of Consulting and Clinical Psychology, 70, 275-287.

doi: 10.1037/0022-006X.70.2.275

Terrace, H. S., \& Metcalfe, J. (Eds.)(2005). The missing link in cognition. Origins of selfreflective consciousness. New York, NY: Columbia University.

Varela, F., Thompson, E., \& Rosch, E. (1991). The embodied mind: Cognitive science and human experience. Cambridge, MA: MIT Press.

Wolman, R. N., \& Kozmová, M. (2007). Last night I had the strangest dream: Varieties of rational thought processes in dream reports. Consciousness and Cognition, 16(4), 838-849. 
doi: 10.1016/j.concog.2006.09.009

Wu, J. K. F., \& Watkins, D. (2006). Testing competing factor models underlying the Private Self-Consciousness Scale with Chinese adolescents. Social Behavior \& Personality, 34(10), 12451258. 


\section{Footnotes}

${ }^{1}$ A discussion of the precise relationship between metacognitive and executive skills is beyond the scope of this paper. For present purposes, we consider metacognitive skills to be a subset of executive functions - we would not claim the two terms can be used inter-changeably (see Ardila, 2008; Fernandez-Duque et al., 2000; Shimamura, 2008, for helpful discussions of the relationship between metacognition and executive functions).

${ }^{2}$ There is a trade-off between sampling metacognition during ongoing experience, and sampling metacognition following as brief a delay as possible. Whereas the former approach invites greater impact of the measure itself on working memory (Baars, 1988), the latter invites greater influence of episodic and constructive memory processes (Horton, Conway, \& Cohen. 2007). One criterion for empirical studies of metacognition, according to Nelson (1996), is knowledge and, ideally, control over the stimulus conditions. The question arises, therefore, as to whether it is feasible to investigate metacognition across states or under conditions where it is otherwise impossible to control the stimulus conditions or to have participants report immediately on their experience (e.g., drug-induced states). In the case of sleep, for example, we would likely never/rarely be able to compare the participant's report or self-assessment with the original ('manipulated') situation. Thus, it is true we could not determine how or whether the individual distorted aspects of the 'original' subjective experience during sleep. Yet, to the extent that recollection of experience is an aspect of metacognitive processing (Leonesio, 2008), efforts to investigate metacognition across states such as sleep and waking have merit, in spite of the inherent methodological limitations)(see Domhoff, 2003; Kahan \& Horton, in press, for further discussion of the methodological challenges in dream science). 
${ }^{3}$ We also conducted principal components analysis with promax rotation, an oblique rotation that allows for correlations among the factors. The same factor structure emerged. Further, the factors accounted for the same proportion of variance in both analyses.

${ }^{4}$ Means and SDs for the four KIMS subscales (Observe, Describe, Act with Awareness, Accept without Judgment) in the present study ( $N=176)$ were, respectively: 37.62 (7.36); 27.91 (5.62); 29.06 (5.39); 29.86 (6.26). Participants’ mean scores for the KIMS subscales were remarkably similar to those reported by Baer et al. for their two student samples ( $N s=205,215$, respectively) (2004, Table 4, p. 203). Also consistent with Baer et al.’s findings, the present findings showed no statistically significant differences between males and females on any of the KIMS subscales. The consistency of the present KIMS results with the findings of Baer et al. further reinforces the integrity of the present data set.

${ }^{5}$ In the present study, 57 males and 118 females completed the SC scale. Mean scores for the three subscales (PrSC, PublSC, SocAnx) for males were, respectively, 26.19, 18.05, 11.95. Mean subscale scores for females were, respectively, 25.6, 19.0, 13.3. Consistent with Fenigstein et al., mean subscale scores did not differ for males and females, as revealed by one-way analysis of variance $(p s>.05)$. In the present study, overall means for the three subscales were, respectively, 25.93, 18.49, and 12.64 . These means are strikingly similar to the overall means reported by Fenigstein et al.: 26.25, 19.1, and 12.65 (computed from Table 2, p. 525). The pattern of intercorrelations among subscales reported by Fenigstein et al. (Table 3, p. 525) was replicated in the present study: PrSC and Publ SC were strongly related, as were PublSC and SocAnx; PrSC and SocAnx were not related (see Table 2).

${ }^{6}$ We did not find a relationship between the MACE and SCS PublSC. It is possible that the MACE factor that emphasizes monitoring the self in relation to external conditions (Monitoring 
External Environment) entails less self-focused attention than does the PublSC scale.

Interestingly, the MACE question most directly based on the PublSC sub-scale ("Were you concerned about the impression you made, how you looked or how you appeared to others?”)

loaded with other questions emphasizing monitoring inner experience, notably feelings (Table 2). The lack of convergence between MACE and SCS PublSC is less surprising when we recall that the Self-Consciousness Scale is a measure of presumably stable individual differences in selffocused attention whereas the MACE is a measure of metacognitive activities associated with current subjective experience. 
Table 1

Factor Structure of the Metacognitive, Affective, Cognitive Experience (MACE) Questionnaire

\begin{tabular}{|c|c|c|c|}
\hline \multirow[b]{2}{*}{ Item Number and Content } & \multicolumn{3}{|c|}{ Factor Loadings } \\
\hline & 1 & 2 & 3 \\
\hline \multicolumn{4}{|l|}{ Self-regulation } \\
\hline $\begin{array}{l}\text { 1. Did you choose between two or more options? } \\
\text { (e.g., I chose to finish my homework instead of going out to the movies) }\end{array}$ & .47 & .38 & -.17 \\
\hline $\begin{array}{l}\text { 4. Did you focus for a period of time on accomplishing a particular task? } \\
\text { (e.g., I looked all over for my keys) }\end{array}$ & .78 & .03 & .13 \\
\hline $\begin{array}{l}\text { 5. Did you experience any unusual difficulty in accomplishing anything } \\
\text { you were trying to do? (e.g., My tennis serve is usually good, but for } \\
\text { some reason I couldn't get the ball over the net) }\end{array}$ & .71 & -.16 & .12 \\
\hline $\begin{array}{l}\text { 9. Did you think about what you were doing? } \\
\text { (e.g., I was thinking I need to be careful not to spill the boiling pot) }\end{array}$ & .68 & .30 & .19 \\
\hline \multicolumn{4}{|l|}{ Monitoring the external environment } \\
\hline $\begin{array}{l}\text { 2. Did you comment to yourself about any person or event? } \\
\text { (e.g., I said to myself "that person looks like my sister") }\end{array}$ & .07 & .60 & .35 \\
\hline $\begin{array}{l}\text { 3. Did something or someone suddenly capture your attention? } \\
\text { (e.g., I heard a scream behind me and I turned around to see what happened) }\end{array}$ & .05 & .72 & -.18 \\
\hline $\begin{array}{l}\text { 10. Did you think about what was happening around you? } \\
\text { (e.g., I thought about how people seemed to be running in all directions at once) }\end{array}$ & .02 & .69 & .30 \\
\hline \multicolumn{4}{|l|}{ Monitoring the internal environment } \\
\hline $\begin{array}{l}\text { 6. Were you concerned about the impression you made, how you looked } \\
\text { or how your appeared to others? (e.g., I was afraid I'd seem foolish if I } \\
\text { asked a question) }\end{array}$ & .04 & -.16 & .79 \\
\hline $\begin{array}{l}\text { 7. Did you feel any emotions during the experiences? } \\
\text { (e.g., I felt angry) }\end{array}$ & .22 & .26 & .56 \\
\hline $\begin{array}{l}\text { 8. Did you think about your own thoughts or feelings? } \\
\text { (e.g., I thought about the intensity of the jealousy I was feeling) }\end{array}$ & .10 & .35 & .46 \\
\hline Percentage of variance accounted for & 27 & 14 & 11 \\
\hline
\end{tabular}


Intercorrelations Among Metacognition, Affective, Cognitive Experience (MACE) Subscales, Self-Consciousness (SC) Subscales, Kentucky Inventory of Mindfulness (KIMS) Scales, Decentering Scale (DC), and the Cognitive Subscale of the Subjective Experiences Rating Scale (SERS)

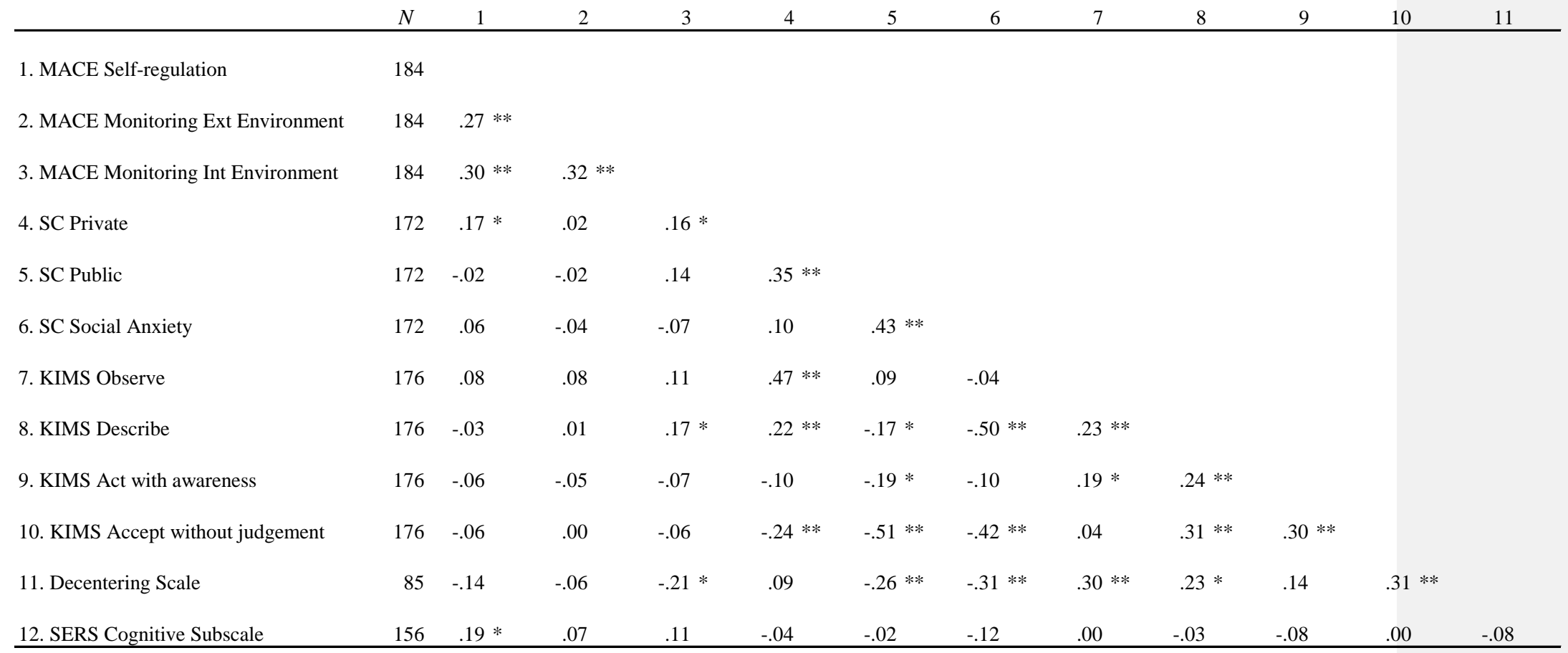

${ }^{*} p<.05 ;{ }^{* *} p<.01$. 
Table 3

Intercorrelations Among Metacognition, Affective, Cognitive Experience (MACE) Subscales and Individual Questions on the Subjective Experiences Rating Scale (SERS)

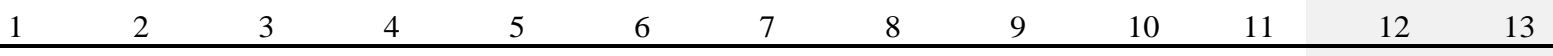

\section{MACE Self-regulation}

2. MACE Monitoring Ext Environment

$.27 * *$

3. MACE Monitoring Int Environment

$.30 * * \quad .32 * *$

4. SERS Thinking

$.35 * * \quad .14 \quad .15$

5. SERS Planning

6. SERS Imagining

$\begin{array}{llll}.14 & -.02 & .06 & .55 * *\end{array}$

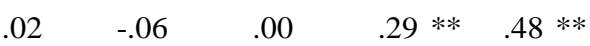

7. SERS Evaluating

$\begin{array}{lllll}.23 * * & -.01 & .10 & .53 * * & .59 * * \quad .41 * *\end{array}$

8. SERS Remembering

$\begin{array}{lllllll}.00 & -.06 & .05 & .27^{* *} & .38^{* *} & .52 * * & .36 * *\end{array}$

9. SERS Feeling

10. SERS Talking

$\begin{array}{lllllllll}.09 & -.05 & .09 & .43 * * & .33^{* *} & .36 * * & .38 * * & .46 * *\end{array}$

$\begin{array}{llllllllll}.01 & .00 & -.02 & .14 & .27 * & .21 * * & .09 & .22 * * & .27 * *\end{array}$

11. SERS Listening

$\begin{array}{lllllllllll}.13 & .10 & .04 & .31 * * & .26 * * & .22 * * & .31 * * & .21 * * & .37 * * & .52 * *\end{array}$

12. SERS Attending Outward

$\begin{array}{lllllllllllll}.13 & .26 * * & .09 & .22 * * & .22 * * & .19 * & .22 * * & .14 & .20 & * & .38 * * & .44 * *\end{array}$

13. SERS Attending Inward

$\begin{array}{llllllllllllllll}.25 * * & .00 & .16 * & .50 * * & .36 * * & .27 * * & .46 * * & .41 * * & .56 * * & .20 * & .37 * * & .28 * *\end{array}$

$* p<.05 ; * * p<.01$ 


\section{Figure Caption}

Figure 1. Illustration of the hierarchical organization of meta-level and object-level, and the hypothesized flow of information in the metacognition. 


\section{Appendix}

\section{Metacognitive, Affective, Cognitive Experiences (MACE) Questionnaire} The MACE. ${ }^{a}$

Instructions: Please answer the following questions with reference to experiences you had during your dream (i.e. the dream you just recorded). First, circle either YES or NO; then, if you circled YES, briefly describe the relevant incident.

1. Did you choose between two or more options? (e.g. I chose to finish my homework instead of going out to the movies)

If YES, please describe:

2. Did you comment to yourself about any person or event? (e.g. I said to myself "that person looks like my sister"; I said to myself “it looks like it might rain”)

If YES, please describe:

3. Did something or someone suddenly capture your attention? (e.g. I heard a scream behind me and I turned around to see what happened)

If YES, please describe:

4. Did you focus for a period of time on accomplishing a particular task? (e.g. I looked all over for my keys)

If YES, please describe:

5. Did you experience any unusual difficulty in accomplishing anything you were trying to do? (e.g. My tennis serve is usually good, but for some reason I couldn't get the ball over the net)

If YES, please describe:

YES NO

6. Were you concerned about the impression you made, how you looked or how you appeared to others? (e.g. I was afraid I'd seem foolish if I asked a question) $\quad$ YES NO

If YES, please describe:

7. Did you feel any emotions during the experiences? (e.g. I felt angry)

YES NO

If YES, please describe

8. Did you think about your own thoughts or feelings? (e.g. I thought about the intensity of the jealousy I was feeling)

If YES, please describe:

9. Did you think about what you were doing? (e.g. I was thinking I need to be careful not to spill the boiling pot)

$\mathrm{NO}$

If YES, please describe:

10. Did you think about what was happening around you? (e.g. I thought about how people seemed to be running in all directions at once)

If YES, please describe:

${ }^{a}$ For permission to use this instrument, please contact tkahan@scu.edu 
